\title{
Multi-Way Optimal Control of a Benchmark Fed-Batch
}

\section{Fermentation Process}

\author{
Hongwei Zhang and Barry Lennox* \\ Control Engineering Research Group, School of Engineering, \\ The University of Manchester, Manchester, UK
}

\begin{abstract}
A novel optimal control algorithm is presented for application to batch processes. The algorithm utilises a linear model of the process that is identified using multi-way partial least squares. This linear model is used within an optimal control law to regulate the productivity of the batch by manipulating the substrates that are fed into the fermentation vessel. The proposed control algorithm is successfully applied to a benchmark fed-batch fermentation process and its performance compared with alternative control algorithms that have been applied to this application in the past. The proposed controller is found to compare favourably with these alternative algorithms.
\end{abstract}

Keywords: Fed-batch fermentation, partial least squares, optimal control

\subsection{Introduction}

The ability to control a fed-batch fermentation process to maximise product yield is of great commercial importance in the biotechnology industry. Such processes represent a very real challenge to control engineers as they possess many characteristics that make them particularly difficult to control. These characteristics include dynamic behaviour that is nonlinear, highly complex and unpredictable and the fact that product concentrations are difficult to measure on-line and may only be available through off-line laboratory assay. 
To stimulate research into the development of advanced controllers for application to fedbatch processes, the Biotechnological Control Forum developed a benchmark simulation of such a system and invited researchers to apply identification and optimal control systems to it (Leigh, 1998). The simulated process, which is provided in scrambled source code, is representative of an industrial fed-batch process containing variations in the initial conditions within the fermenter, unmeasured disturbances and physical constraints on several of the process variables.

In response to the invite by the Biotechnological Control Forum to investigate this process, McKay et al (1998) applied a genetic programme to help develop a non-linear model of the system. The accuracy of the model was found to be very good, particularly for short-term prediction during the batch, which is what would typically be required in an optimal control algorithm. In a further investigation, Becerra and Roberts (1998) applied a control algorithm, termed Dynamic Integrated System Optimisation and Parameter Estimation (DISOPE) to the process. Despite using a linear model within this controller, the DISOPE algorithm performed very well, with improvements of over $21 \%$ being made to the final productivity of the process.

In this paper multi-way partial least squares (MPLS) is used to develop a soft-sensor that is capable of predicting biomass and product concentrations within the fermenter. Recent investigations have demonstrated the suitability of using MPLS as a soft-sensor in industrial fed-batch fermentation systems (Lennox et al, 2001 and Lakshminarayanan et al, 1996). Despite the fact that MPLS is a linear approach, the results in this paper indicate that its ability to model the fed-batch benchmark system compares favourably with the non-linear approach reported by McKay et al (1998).

Following its development, the MPLS model is integrated within an optimal control strategy that is used to regulate the feedrates of the two substrates in the process to maximise productivity. Test results indicate that although the structure of the proposed control system is 
very simple, it provides improved performance when compared with the much more complex DISOPE algorithm proposed by Becerra and Roberts (1998).

The paper continues in section 2 with a description of the simulated benchmark process. This is followed in section 3 with an overview of the MPLS technology and a summary of the results obtained when the approach was applied, as a soft-sensor, to the benchmark fed-batch fermentation process. Section 4 then describes how the developed MPLS model can be integrated within an optimal control strategy. Results collected when the proposed controller is applied to the benchmark fed-batch process are also provided in section 4. Finally a list of conclusions arising from this investigation is provided in section 5.

\subsection{Problem Description}

The benchmark process represents a fed-batch fermentation process that produces a secondary metabolite as the product. For growth and production the metabolite requires two substrate feeds, $S_{1}$ and $S_{2}$. During each batch, on-line measurements of the substrate feedrates and concentrations, $F_{1}$ and $F_{2}$ and $S_{1}$ and $S_{2}$ respectively, biomass concentration, $X$, product concentration, $P$ and reactor volume, $V$, are available. As with an industrial fermentation process the initial conditions of each batch are variable, the process is subject to unmeasured disturbances and noise is present on all measurements. Other process environment variables, such as temperature and $\mathrm{pH}$, are assumed to be kept constant at their optimum values.

To be representative of an industrial application, the authors of the simulation provided the following information:

1. A black-box model in scrambled source code that simulates the plant.

2. Typical substrate feed rate profiles which can be used in the simulation to generate input/output data. 
3. Specifications of the constraints, which are defined as:

$$
\begin{aligned}
& 0 \leq F_{1} \leq 50 \text { units } \\
& 0 \leq F_{2} \leq 50 \text { units } \\
& V \leq 4000 \text { units }
\end{aligned}
$$

\subsection{Soft-sensing using Multi-way Partial Least Squares}

\section{$\underline{\text { 3.1 Partial Least Squares (PLS) }}$}

PLS is a system identification tool that is capable of identifying the relationships between cause $(\mathbf{X})$ and effect $(\mathbf{Y})$ variables. The advantage that this approach offers over more traditional identification techniques, such as ordinary least squares, is that it is able to extract robust models even in applications involving large numbers of highly correlated and noisy process variable measurements.

The approach works by selecting factors of cause variables in a sequence that successively maximises the explained covariance between the cause and effect variables. Given a matrix of cause data, $\mathbf{X}$, and effect data, $\mathbf{Y}$, a factor of the cause data, $\mathbf{t}_{k}$, and effect data, $\mathbf{u}_{k}$, is evaluated, such that:

$$
\mathbf{X}=\sum_{k=1}^{n p<n x} \mathbf{t}_{k} \mathbf{p}_{k}^{T}+\mathbf{E} \text { and } \mathbf{Y}=\sum_{k=1}^{n p<n x} \mathbf{u}_{k} \mathbf{q}_{k}^{T}+\mathbf{F}
$$

where $\mathbf{E}$ and $\mathbf{F}$ are residual matrices, $n p$ is the number of inner components that are used in the model and $n x$ is the number of causal variables. $\mathbf{p}_{k}$ and $\mathbf{q}_{k}$ are referred to as loading vectors.

These equations are referred to as the outer relationships. The vectors $\mathbf{t}_{k}$ are mutually orthogonal. These vectors and $\mathbf{u}_{k}$ are selected so as to maximise the covariance between each 
pair, $\left(\mathbf{t}_{k}, \mathbf{u}_{k}\right)$. Linear regression is performed between the $\mathbf{t}_{k}$ and the $\mathbf{u}_{k}$ vectors to produce the inner relationship, such that:

$\mathbf{u}_{k}=b_{k} \mathbf{t}_{k}+\varepsilon_{k}$

where $b_{k}$ is a regression coefficient, and $\varepsilon_{k}$ refers to the prediction error. The PLS method provides the potential for a regularised model through selecting an appropriate number of latent variables, $\mathbf{u}_{k}$ in the model $(n p)$. The number of latent variables is typically made through the use of cross validation.

For further details of the PLS algorithm, the reader is referred to Geladi and Kowalski (1986).

\subsection{Multi-way Partial Least Squares (MPLS)}

PLS is a linear tool, which unfortunately limits its effectiveness when applied to non-linear fed-batch processes. Two options exist for improving the capabilities of PLS when applied to fed-batch systems. The first is to develop non-linear counterparts to PLS and the second is to transform the fed-batch data in such a way as to remove the non-linear characteristics. Although non-linear PLS techniques exist (Qin, 1992), the transformation of batch data has proved to be a more effective option and has been adopted in this investigation. The most common form of data transformation, termed multi-way PLS, was initially proposed by Nomikos and MacGregor (1994). Since then other researchers have adopted the approach and applied it to a variety of processes. For example, Gallagher et al (1996) applied the technique to monitor nuclear waste storage vessels and Lennox et al (2001) and Lakshminarayanan et al (1996) investigated the detection of faults in fed-batch fermentation processes.

In a fed-batch process, the cause and effect data can be thought of as being in two 3dimensional arrays of size $n b \times n x \times m x$ and $n b \times n y \times m y$, where $n b$ is the number of batches 
for which data is available, $n x$ and $n y$ are the number of cause and effect variables respectively and $m x$ and $m y$ are the number of observations of the cause and effect variables respectively that are made during a batch. Unfortunately, PLS requires that the cause and effect arrays be two-dimensional. To address this problem the three-dimensional arrays are recast into two-dimensional arrays in a process referred to as unfolding. The concept of unfolding is illustrated in figure 1. The original data arrays are unfolded into a cause variable array, $\boldsymbol{X}$, of size $n b \times(n x * m x)$ and an effect variable array of size $n b \times(n y * m y)$. It should be noted that the number of observations made of the cause variables need not be equal to the number of observations made for the effect variables. In fact, it is relatively common for there to be a single effect measurement made during a batch. This measurement being the final product quality taken at the end of the batch. Following the unfolding of the data, it is then possible to apply PLS to the data in the conventional manner.

The subsequent use of this model on-line poses the problem that it is necessary to know the values of all process measurements through to the end of the batch, since the unfolded array contains the measurements of each of the variables throughout the duration of the batch. This means that with the exception of the end point of the batch, it is necessary to estimate the future values of all the measured variables. The prediction of future process values is referred to as filling up the array. Of the three methods that were suggested by Nomikos and MacGregor (1994) for filling up the array, Lennox et al (2001) found that the most appropriate method for an industrial fed-batch process was to assume that the values of all process measurements remain at their current offset from the mean trajectory through to the end of the batch. Whilst the most suitable filling up method is likely to be process dependent, this method was also found to be the most appropriate in this work.

\subsection{Application of MPLS to the Benchmark Plant}

To develop a model of the fermentation system it is necessary to collect plant data from a series of reference batches. The original simulation that was provided by the Biotechnological 
Control Forum operated with exponential feedrates for the two substrates. These feedrates were defined as follows:

$$
F_{1}(t)=10+\frac{25}{1+e^{5-0.1 t}} ; \quad F_{2}(t)=3.5-\frac{3.5}{1+e^{10-0.15 t}}
$$

In this paper we refer to these as the nominal feedrates.

Ideally the reference batches should contain data collected during full excitation of the process, for example PRBS sequences could be applied to the feedrates of the two substrates. Unfortunately, it is difficult to obtain the necessary permission to perform thorough plant tests since operating the process under such conditions will typically result in a poor quality batch, which is unlikely to be acceptable. Therefore in this application the models were developed using data collected from batches that operated under nominal conditions, or conditions close to nominal.

Data from a total of 30 batches, referred to as reference batches, were used to develop the MPLS model. Each batch was allowed to run for 200 time units, with the sample time set to 0.25 time units. The MPLS model was calculated using 20 of the reference batches, with the remainder (testing batches) being used to assess the performance of the model. The model contained 3 latent variables.

The accuracy of this model for product and biomass prediction, over the 10 testing batches, is displayed in figures 2 and 3 respectively. In each figure the top, middle and bottom graphs represent the 25, 50 and 100 step-ahead predictions respectively and the grey and black lines represent the actual and predicted product and biomass measurements respectively. The vertical dashed lines in these figures indicate when one batch ends and the next begins. The 
numbers on these figures provide a numerical measure of the accuracy of the model. This measure, the average squared prediction error (ASPE), being defined as:

$A S P E=\frac{\sum_{k=1}^{800}\left[\hat{y}_{k}-y_{k}\right]^{2}}{800}$

where $y_{k}$ is the actual product / biomass concentration and $\hat{y}_{k}$ is the predicted product / biomass concentration. There are 800 sample points during the batch, hence the division by 800.

Figures 2 and 3 demonstrate that the accuracy of the model is very good and would appear to compare favourably with the results reported by McKay et al (1998).

In addition to predicting the concentration of the biomass and product during the batch, it is also helpful to have an indication of the expected yield from the batch. This can be achieved by using the MPLS model to predict the concentration of product and biomass that is expected to be present within the fermentation vessel at the end of the batch. Although each batch was operated for 200 time units, the end-point of the batch was taken as being after 120 time units. The reason for this is that from the reference batches, it is evident that the concentration of product in the fermenter begins to drop after this time. 120 time units could therefore be considered a reasonable point at which the batch should be harvested.

Figures 4 and 5 demonstrate the accuracy of the MPLS model at predicting the end-point concentration of the product and biomass respectively, for the 10 testing batches. The black line in these figures represents the concentration of product and biomass that was actually recorded at the end of the batch. The grey line in the figures represents the end-point concentration that is predicted by the PLS model during the batch. The vertical dashed lines 
indicate when one batch ends and the next begins. These graphs indicate that the prediction accuracy is poor during the initial stages of the batch, but then improves significantly. The reason for the poor accuracy initially is because at the start of the batch most of the data in the unfolded data matrix has been estimated and is therefore less reliable than in the later stages of the batch.

\subsection{Batch Control Using Multi-way Optimal Control}

Based upon the model developed in section 3 there are two possible control strategies that were considered. The first is to use the continuous on-line prediction of product and biomass to develop a control strategy that will attempt to force the product and biomass concentrations to follow a pre-specified trajectory by manipulating the two feed streams. The second possible control structure is slightly simpler and involves optimising the end-point product concentration by manipulating the two feedrates. Since it is not clear what form any prespecified trajectory for the product and biomass concentrations should take the decision was made to use the end-point controller.

This controller utilises the following cost function:

$$
J=\left[\hat{y}_{t_{\text {end }}}-w_{t_{\text {end }}}\right]^{2}
$$

where $\hat{y}_{t_{\text {end }}}$ is equal to the prediction made by the MPLS model of the product concentration at the end point of the batch and the set-point, $w_{t_{\text {end }}}$, is the desired final product concentration.

At each sampling instant, $t_{s}$, the controller identifies what changes should be made to the current value of the two substrate feedrates to minimise the value of equation 1 . In performing its calculations the controller assumes that only a single change is made to each of the two 
substrate feeds, i.e. the feedrates of substrate 1 and 2 at sample time $t_{s}+1$, denoted as $F_{1}\left(t_{s}+1\right)$ and $F_{2}\left(t_{s}+1\right)$ respectively. Following this change the values of these feedrates are assumed to remain at their current offset from nominal conditions for the remainder of the batch. To achieve the optimisation of the cost function defined in equation 1 , the controller utilises a float-encoded genetic algorithm (FGA) (Zhang et al, 2000). The FGA searches in the permissible operating space of the process, subject to the constraints $0 \leq F_{1} \leq 50$ units, $0 \leq F_{2} \leq 50$ units and $V \leq 4000$ units, for a suitable value for $F_{1}\left(t_{s}+1\right)$ and $F_{2}\left(t_{s}+1\right)$ that will minimise equation 1 . This control move is then applied and the calculations repeated at the following sampling instant. The procedure for the FGA is summarised below:

1. One hundred possible values for $F_{1}$ and $F_{2}$ are selected at random from the permissible solution space and encoded as floating-point decimal numbers. These values are termed the population of possible solutions.

2. The cost function, equation 1 , is evaluated for each of the 100 possible values of $F_{1}$ and $F_{2}$.

3. If the finishing criteria is met, which in this case was that either the routine had converged to a minima or the maximum number of iterations had been exceeded, then the optimal values of $F_{1}$ and $F_{2}$ are applied to the process, otherwise continue.

4. Apply standard genetic operations (such as cross-over and mutation) to the population and generate a new population of possible solutions.

5. Return to 2 .

It is standard practice with control systems for there to be a tuning parameter within the controller to ensure that the controller achieves acceptable performance. In this work it was found that the controller was capable of delivering acceptable performance by simply imposing constraints on the incremental values of the two substrate feedrates, $F_{1}$ and $F_{2}$. Without this constraint the controller tended to make excessive control moves which had a 
detrimental effect on micro-organism growth. For this application the feedrates were prevented from varying by more than $20 \%$ from one sampling instant to the next.

The authors of this benchmark process suggest that the following measure be used when assessing the performance of any proposed controller:

$$
J_{c}=\frac{P^{*} V}{t}
$$

where $P$ is the concentration of product in the fermenter when it is harvested, $V$ is the volume of contents in the fermenter at the end-point of the batch and $t$ is the run length of the batch.

This performance measure indicates that the control system should produce the maximum amount of product, in the minimum length of time.

From the earlier observations made regarding the productivity observed during the reference batches, the run length is assumed to be 120 time units. This value is consistent with the run length used by Becerra and Roberts (1998) in their study. The set-point, $w_{t_{\text {end }}}$, was set to 3500 . Whilst specifying the target end-point concentration does not allow the productivity of the batch to be maximised, based upon the results presented by Becerra and Roberts (1998) a figure of 3500 is believed to be close to the maximum productivity that is achievable from this process. Whilst it may seem appropriate to simply select an extremely large value for $w_{t_{\text {end }}}$ in order to produce the maximum product concentration, this can lead to problems as discussed later in this section. This controller is referred to as MOC1.

Figure 6 shows the product concentration that was produced for 10 separate batches using this control strategy (light grey lines). The bold line in this figure represents the average product 
concentration profile measured during these 10 batches. Although the set-point concentration of 3500 is not quite achieved in each batch, the trajectory of the product concentration does compare favourably with the thick grey line, which represents the average product concentration profile that was produced under nominal feedrate conditions.

The feedrates of the two substrates and the volume measurement within the fermenter, when the process is operated under nominal conditions and using the MOC1 controller, are provided in figure 7. It is clear from figure 7 that there is a marked difference between the feedrate profile of substrate 1 measured under closed loop operation and that measured under nominal conditions. However, the feedrate profile of substrate 2 has changed only slightly. This is to be expected as the authors of the benchmark simulation state that substrate 2 seems to be complementary to substrate 1 . Figure 8 illustrates the improvement in the average production $\left(P^{*} V\right)$ that was recorded during the batches implementing the MOC1 controller compared with that obtained in the reference batches. The average production of biomass produced by $\mathrm{MOC} 1$ is compared in figure 9 with the average produced for the reference batches. It is clear from figure 9 that the proposed controller has had only a limited impact on the production of biomass within the fermenter.

As stated earlier, one of the objectives is to minimise the run length of the batch. To analyse the effect of reducing the run length, the length of the batch was reduced from 120 to 100 time units. Reducing the value of the run length necessitated the reduction in $w_{t_{\text {end }}}$ from 3500 to 3000 . With $w_{t_{\text {end }}}$ set to 3500 , the controller performed poorly. The reason for this is that the MPLS model is linear and whilst the unfolding technique has attempted to remove the major non-linearity in the process, the MPLS model will have greatest accuracy the closer the process lies to nominal conditions. When the set-point is set to 3500 , the resulting controller drives the process well away from the nominal conditions, which has a detrimental effect on the accuracy of the model, and therefore the performance of the controller. As with all model 
based control systems, the performance of this controller will be limited by the quality of the model. Since the model was developed on relatively low production batches, its performance as the process moves away from these conditions will begin to deteriorate, primarily because of the non-linear nature of the process. There are a number of possible solutions that could be adopted to improve the performance of the model, which include replacing the PLS model with a non-linear approach and adapting the PLS model to changing operating conditions. For application to fed-batch systems, such as that considered here, the second approach is believed to be particularly appropriate and will be the subject of future work.

A simple approach that was applied in this work to alleviate the problem when $w_{t_{\text {end }}}$ was set to 3500 , was to specify $w_{t_{\text {end }}}$ to be equal to 3000 . By doing this it is not necessary for the controller to move the operating conditions as far away from nominal than if $w_{t_{\text {end }}}$ was set to 3500 and as a consequence the performance of the controller remains acceptable. This controller is referred to as MOC2.

Figure 10 shows the product concentration obtained over a series of 10 batches (light grey lines) for this controller. The bold line in this figure represents the average product concentration measured during these 10 batches which can be compared with the thick grey line, which represents the average productivity under nominal feedrates. Figure 11 shows the performance of this controller, in terms of average production $\left(P^{*} V\right)$ measured during the batch. As with the earlier controller, MOC1, the performance of this controller, in terms of increased product concentration, is seen to be significantly better than that produced using the nominal feedrates.

Table 1 compares the performance of the two developed controllers with the production capabilities of the process using the nominal feedrates and also the controller developed by Becerra and Roberts (1998). 


\begin{tabular}{lcccc}
\hline Controller & $\begin{array}{c}\text { Run Length } \\
t_{\text {end }}\end{array}$ & $\begin{array}{c}\text { Average Final Product } \\
\text { Concentration }(P)\end{array}$ & $\begin{array}{c}\text { Total Production } \\
\left(P^{*} V\right)\end{array}$ & $\begin{array}{c}\text { Productivity } \\
\left(P^{*} V / t_{\text {end }}\right)\end{array}$ \\
\hline Nominal Feed & 120 & 2320 & $9.25 \mathrm{E}+06$ & $7.71 \mathrm{E}+04$ \\
MOC1 & 120 & 3210 & $1.28 \mathrm{E}+07$ & $1.07 \mathrm{E}+05$ \\
DISOPE & 120 & 2900 & $1.16 \mathrm{E}+07$ & $9.50 \mathrm{E}+04$ \\
Nominal Feed & 100 & 1700 & $5.86 \mathrm{E}+06$ & $5.86 \mathrm{E}+04$ \\
MOC2 & 100 & 2680 & $1.07 \mathrm{E}+07$ & $1.07 \mathrm{E}+05$ \\
\hline
\end{tabular}

Table 1: Controller Performance Assessment

The results summarised in table 1 indicate that the proposed MPLS optimal controller increases productivity by almost $40 \%$ when compared with the productivity resulting from the use of the nominal feedrates. The productivity of the MPLS optimal controller is also $12 \%$ greater than that produced by the DISOPE controller. It is also evident from this table that there is no clear difference in productivity produced by MOC1 and MOC2.

\subsection{Conclusions}

This paper has described the application of an optimal control strategy to a benchmark fedbatch simulation. The optimal control strategy utilises a linear model, developed using multiway partial least squares, which acts as a soft-sensor and provides long-term predictions of product concentration at the end of the batch, to determine suitable substrate feedrates to ensure that productivity of the batch reaches a required level.

The results that are reported in this paper indicate that the proposed soft-sensor and controller compare favourably with those reported in other studies involving this benchmark process. In 
particular, when compared with the nominal operating conditions, provided by the authors of this simulation, the proposed controller was found to improve productivity by almost $40 \%$.

\section{Acknowledgements}

The authors would like to thank EPSRC who have funded this work (grant number: GR/N24858).

\section{References}

Becerra, V.M. and Roberts, P.D., (1998), 'Application of a novel optimal control algorithm to a benchmark fed-batch fermentation process', Transactions of the Institute of Measurement and Control, 20(1), 11-18

Gallagher, N.B., Wise, B.M. and Stewart, C.W. (1996), “Application of multiway principal components analysis to nuclear waste storage tank monitoring", Comp. Chem. Eng., 20S, $739-744$

Geladi, P. and Kowalski, B.R., (1986), "Partial least squares regression: a tutorial”, Anal. Chim. Acta., 185, 1-17

Lakshminarayanan, S., Gudi, R.D., Shah, S.L. and Nandakumar, K. (1996), "Monitoring batch proceses using multivariate statistical tools: extensions and practical issues", Proceeding of IFAC World Congress, San Francisco, 241-246

Leigh, R., (1998), 'Fed-batch fermentation process modelling and control competition', Transactions of the Institute of Measurement and Control, 20(1), 3

Lennox, B., Hiden, H.G., Montague, G.A., Kornfeld, G. and Goulding, P.R., (2001), 'Application of multivariate statistical process control to batch operations', Biotechnology and Bioengineering, 74 (2), 125-135

McKay, B., Sanderson, C.S., Willis, M.J., Barford, J.P. and Barton, G.W., (1998), 'Evolving a hybrid model of a fed-batch fermentation process', Transactions of the Institute of Measurement and Control, 20(1), 4-10 
Nomikos, P. and MacGregor, J.F. (1994), "Monitoring batch processes using multi-way principal component analysis", AIChE Journal, 40 (8), 1361-1375

Qin, S.J. and McAvoy, T.J., (1992), 'Nonlinear PLS modelling using neural networks', Computers and Chemical Engineering, 16 (4), 379-391

Zhang, H., Lennox, B., Goulding, P.R. and Leung, A.Y.T., (2000), 'A float-encoded genetic algorithm technique for integrated optimization of piezoelectric actuator and sensor placement and feedback gains', Smart Materials and Structures, 9 (4), 552-557 


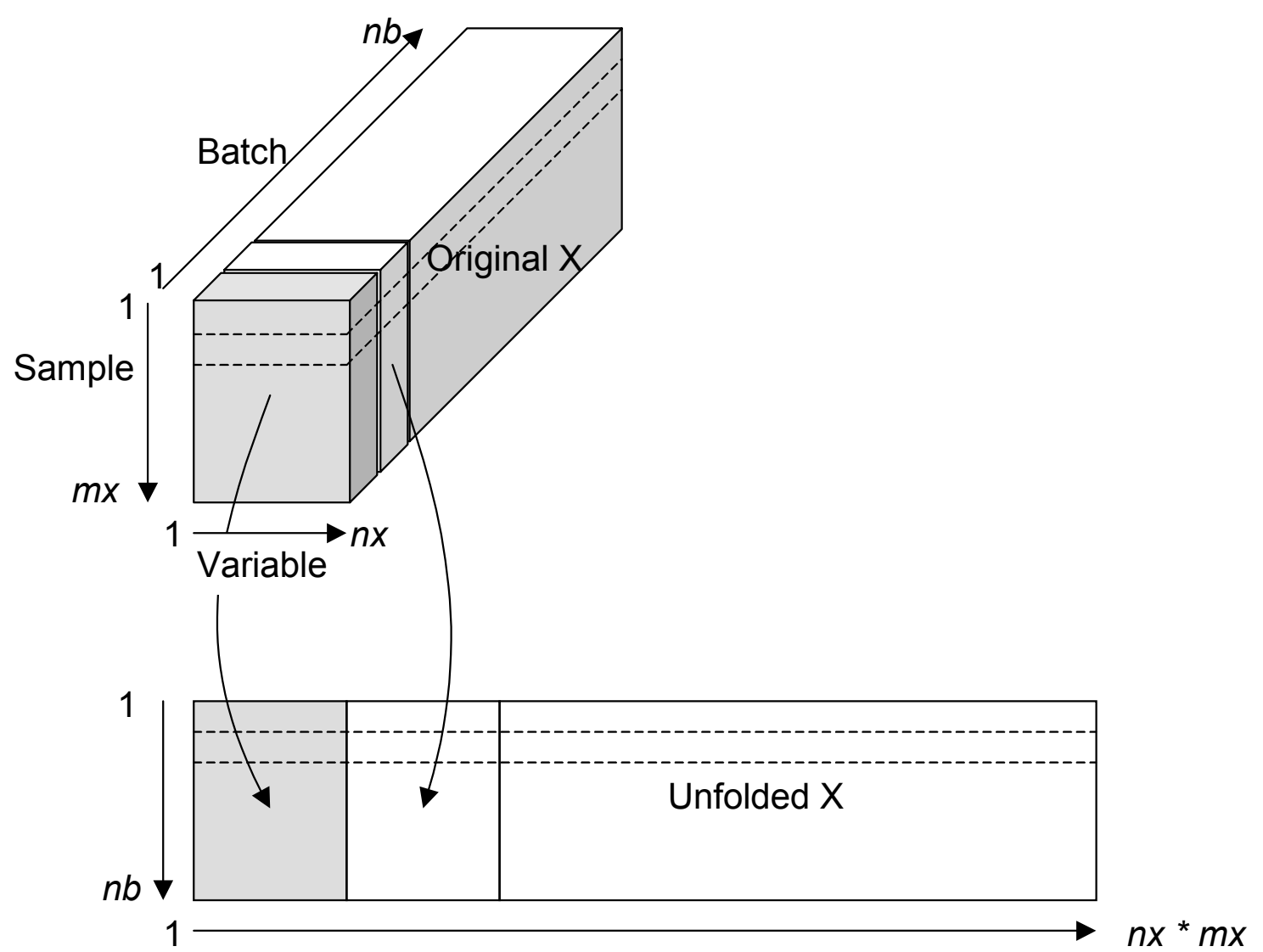

Figure 1: Unfolding 
a) $\mathbf{2 5}$ step-ahead prediction

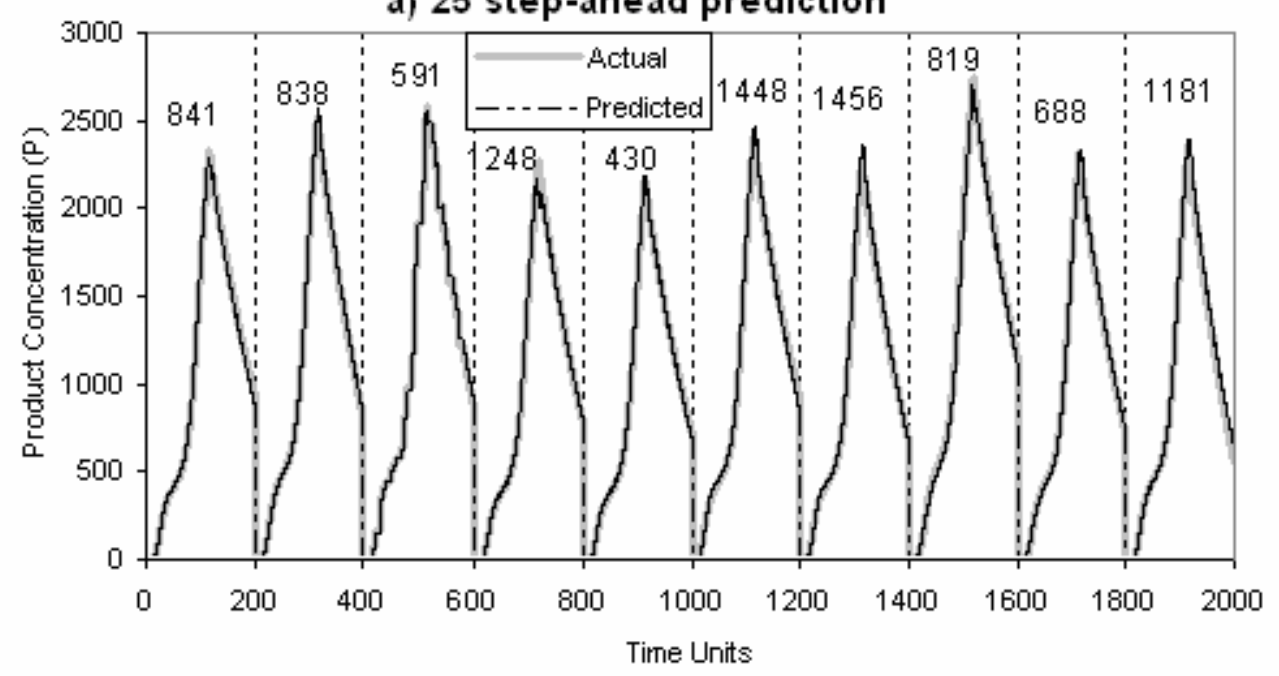

b) $\mathbf{5 0}$ step-ahead prediction

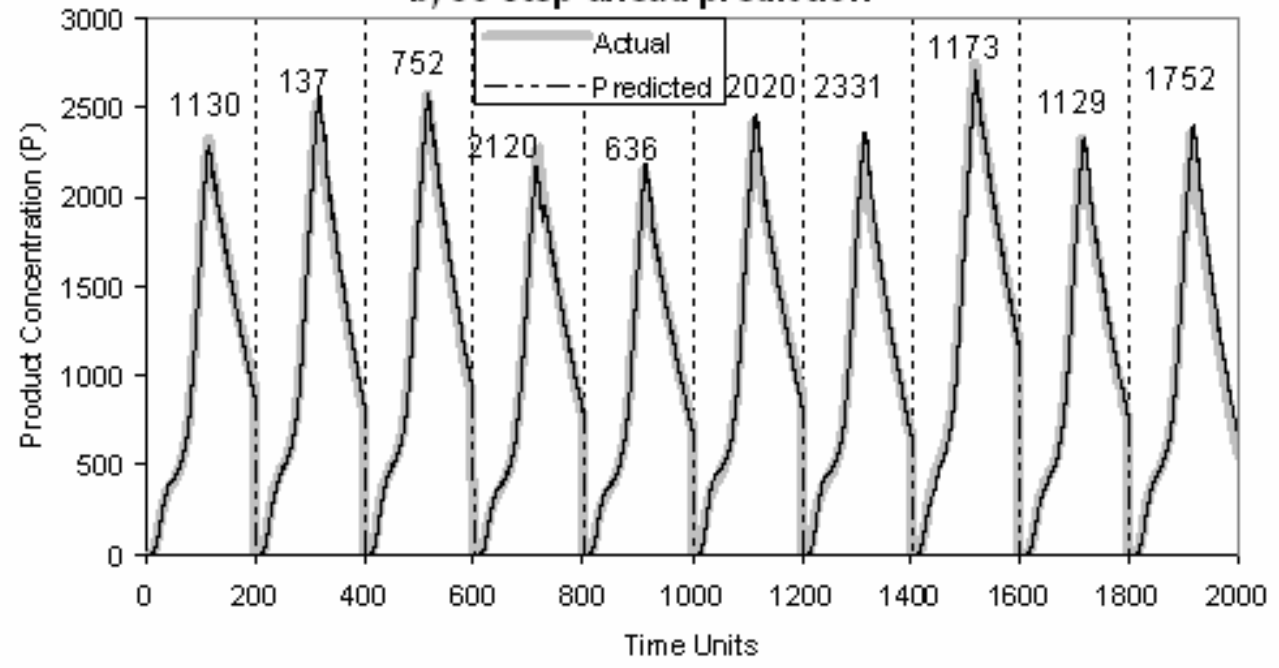

c) 100 step-ahead prediction

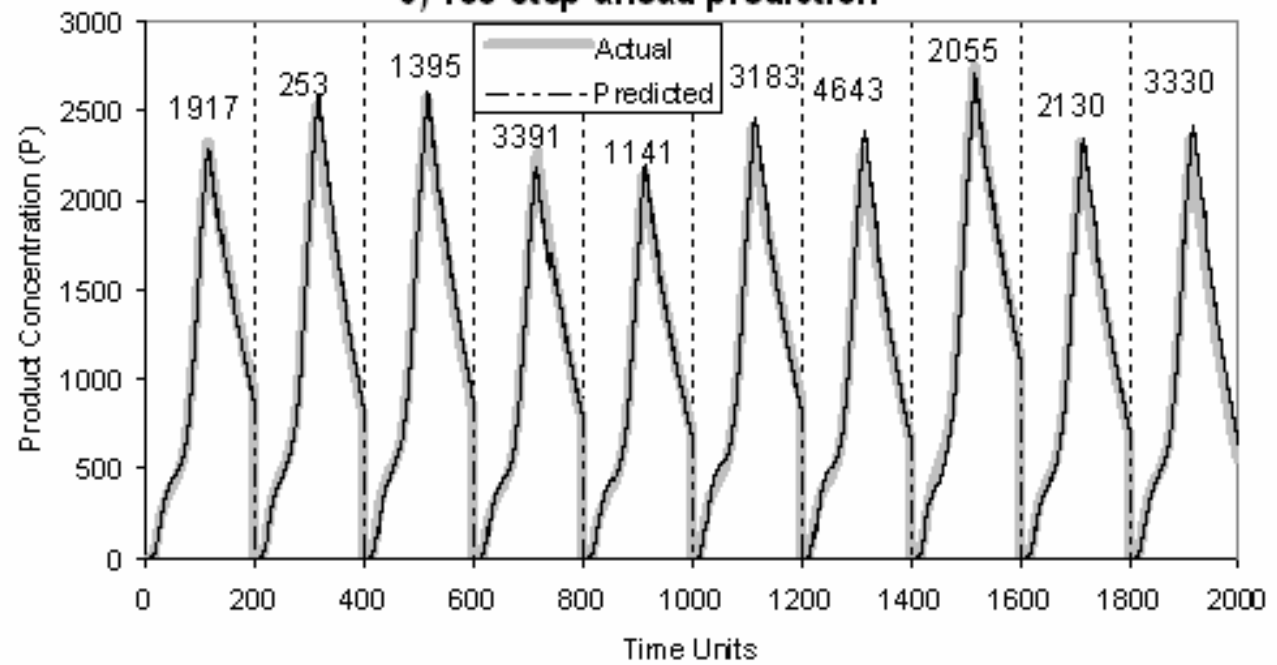

Figure 2: Prediction of product concentration 
a) 25-step ahead prediction
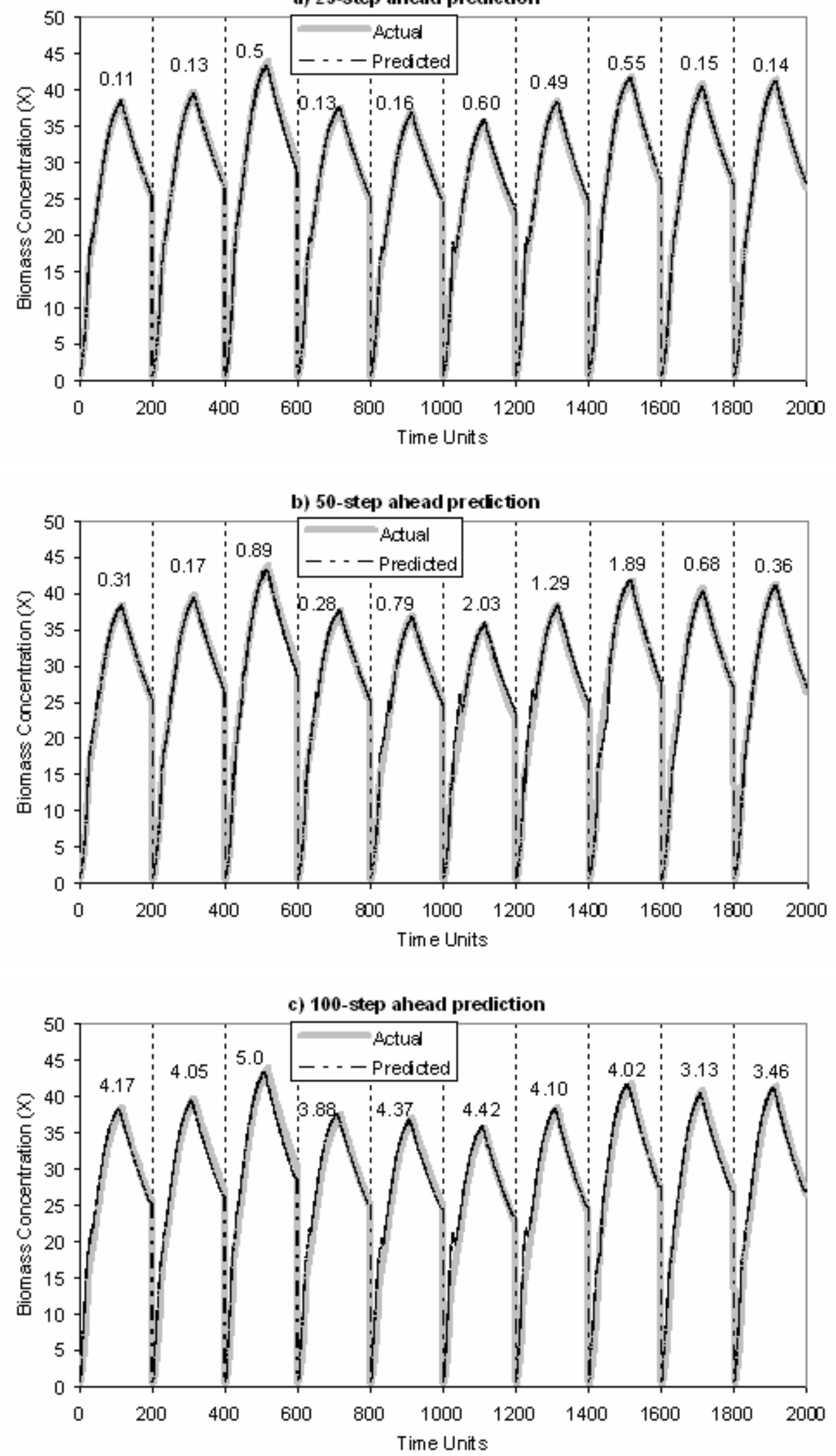

Figure 3: Biomass prediction 


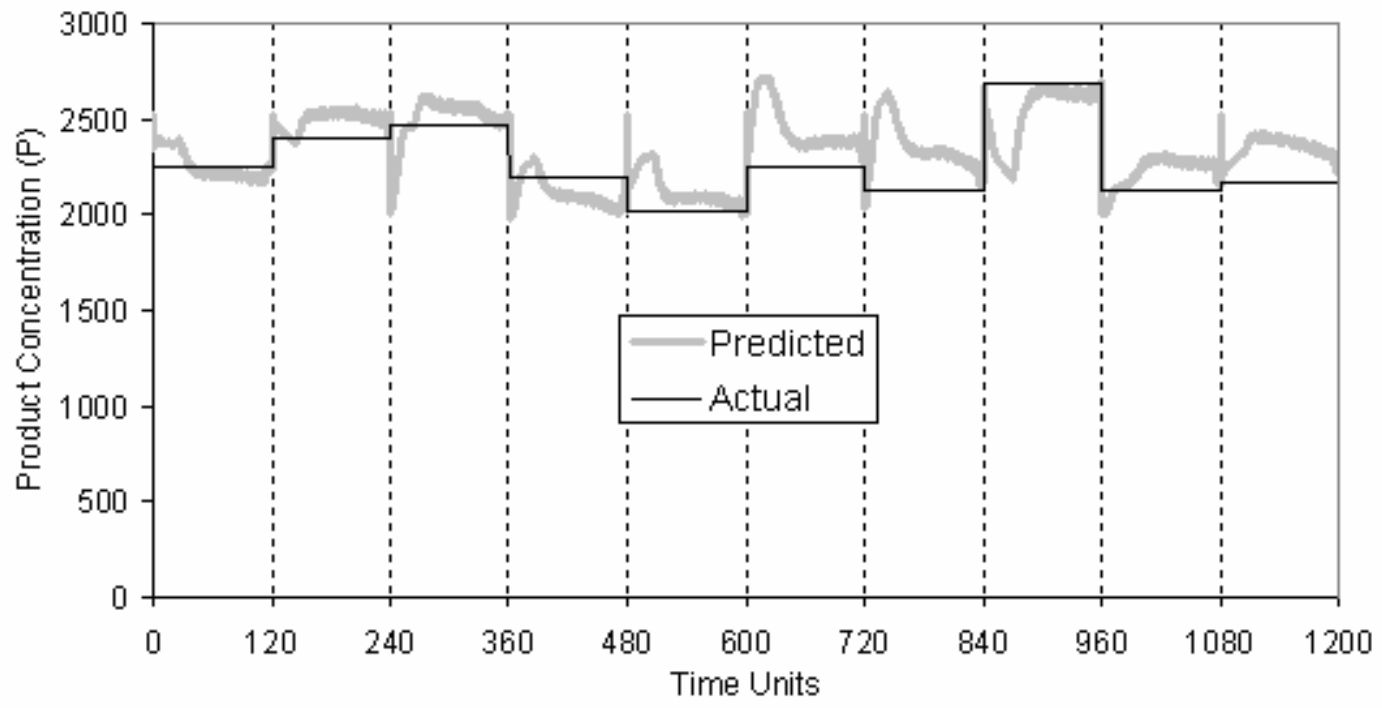

Figure 4: End-point product prediction

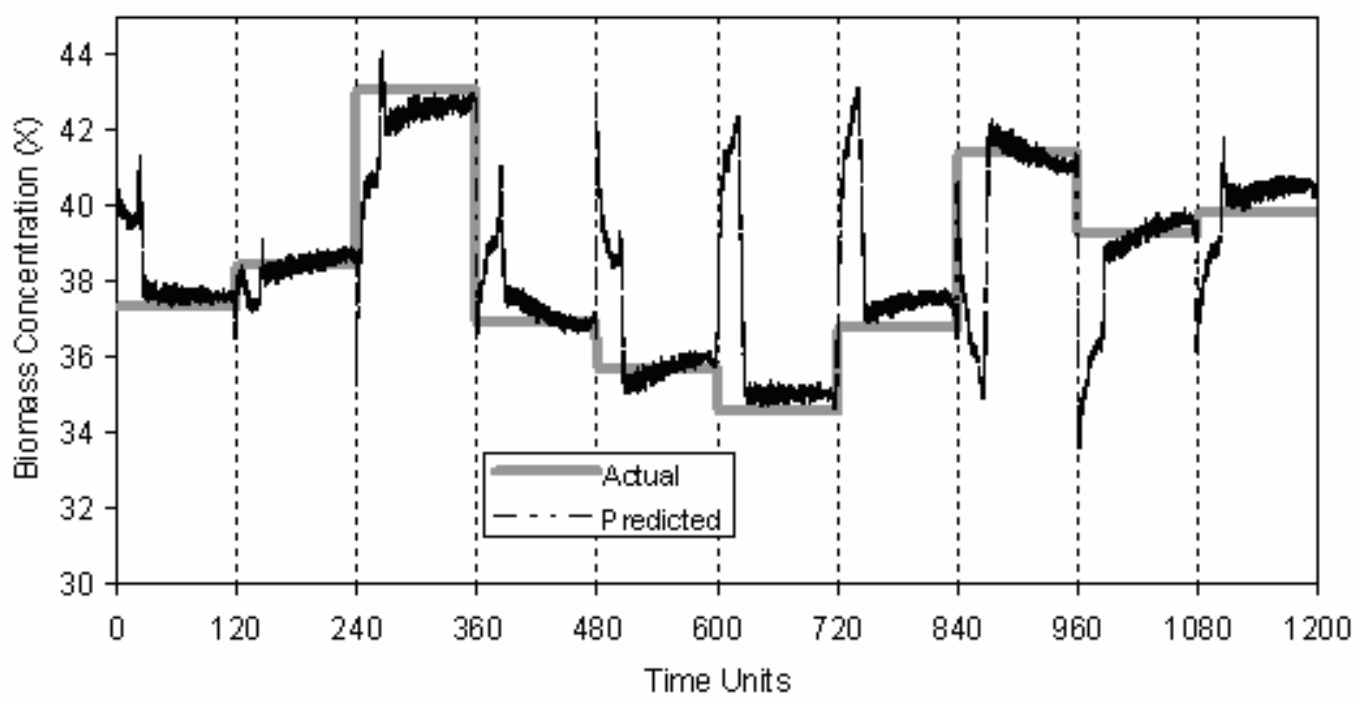

Figure 5: End-point biomass prediction 


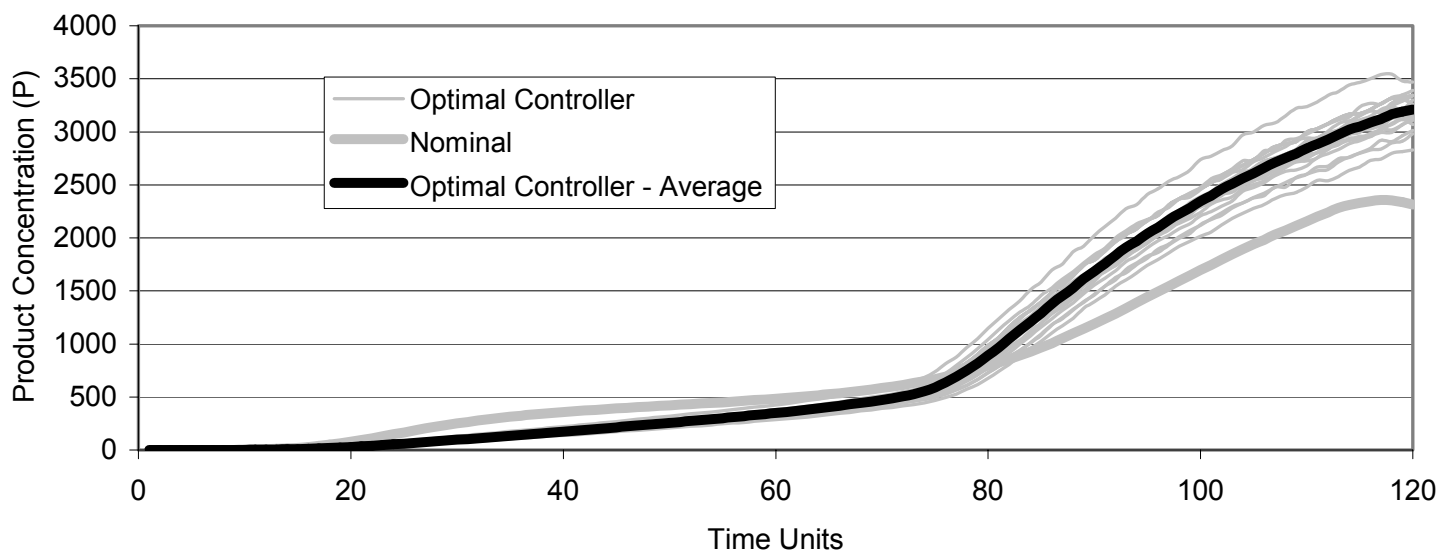

Figure 6: Performance of optimal controller with run length of 120 time units 

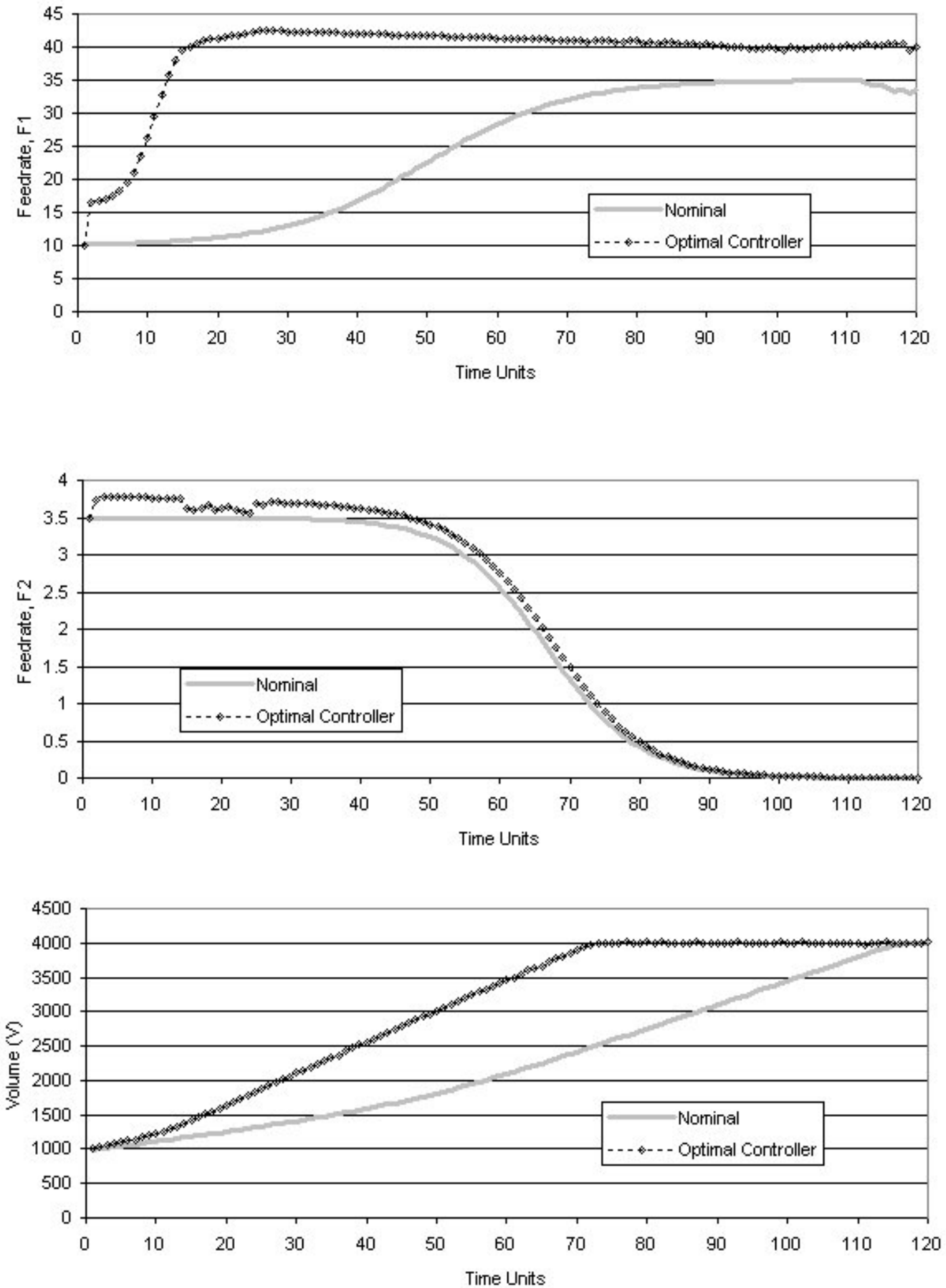

Figure 7: Average Process Conditions During Batch 


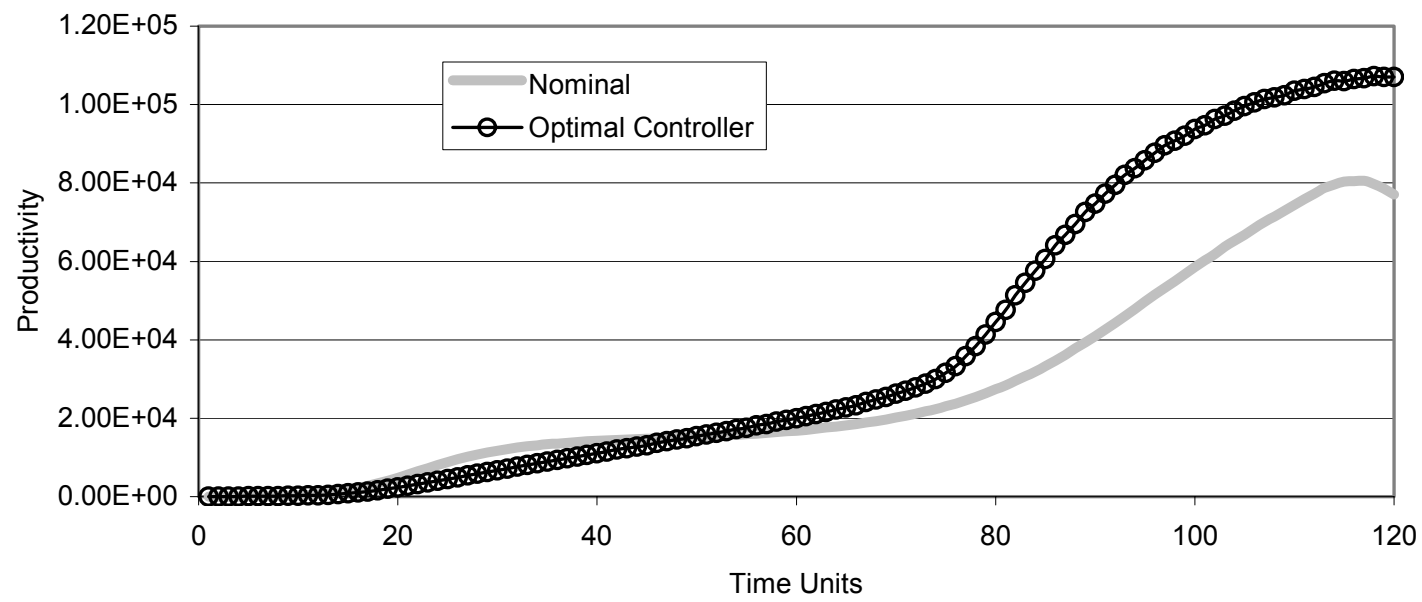

Figure 8: Productivity

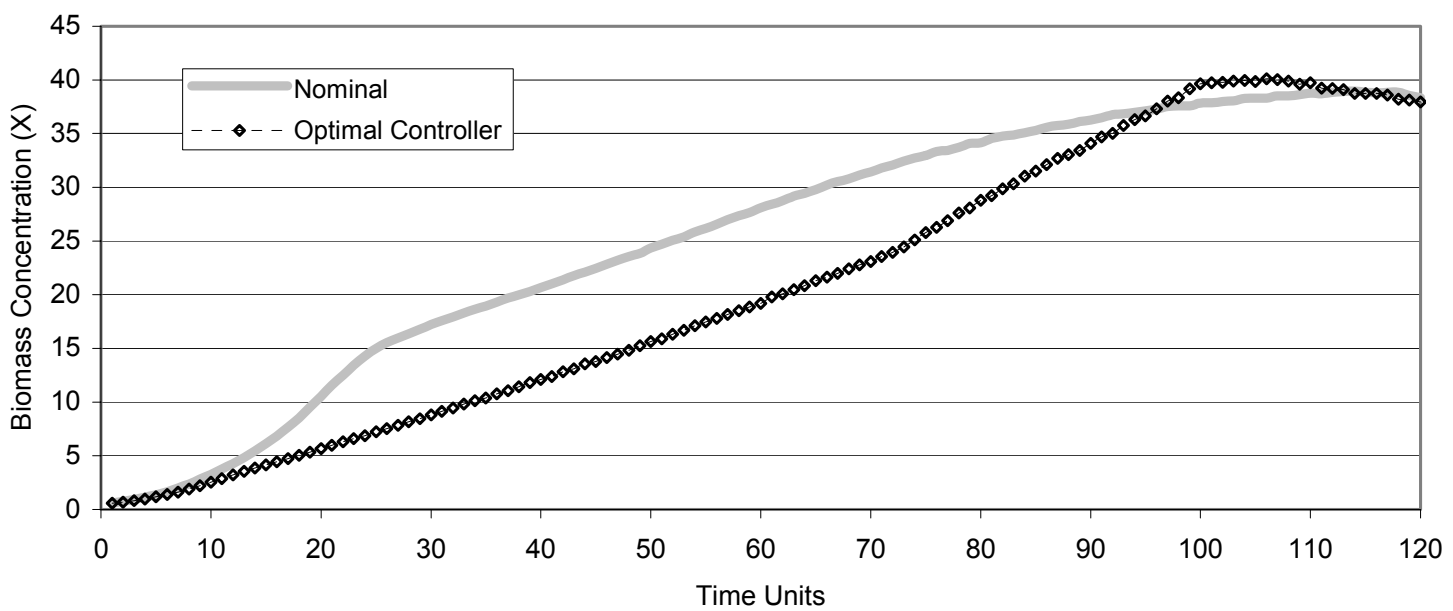

Figure 9: Biomass concentration during the batch 


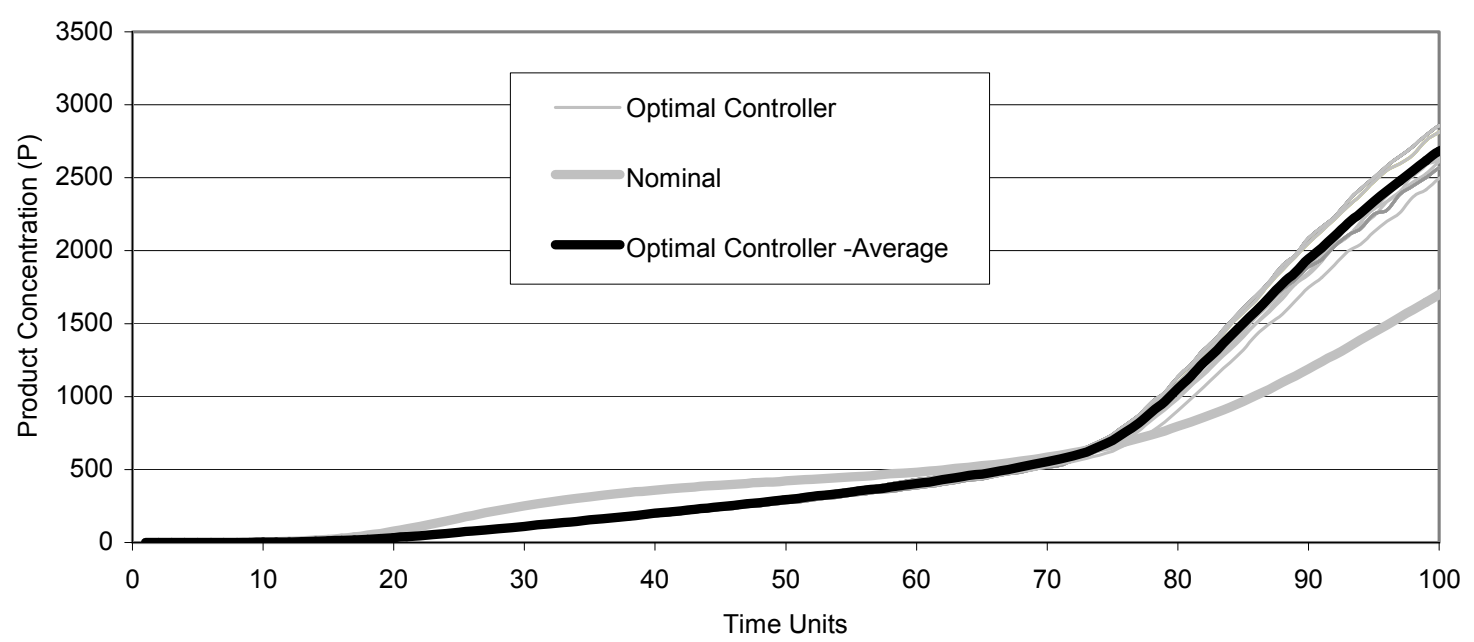

Figure 10: Performance of optimal controller with run length of 100 time units

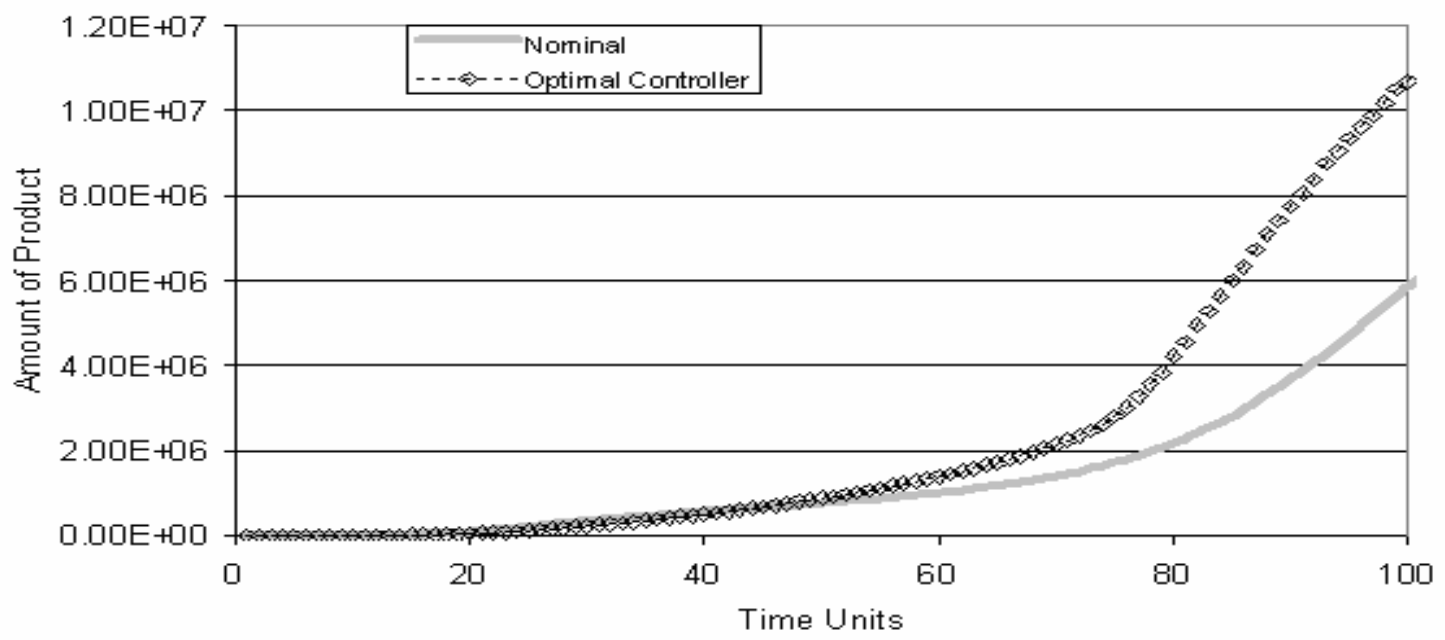

Figure 11: Amount of Product with run length set to 100 time units 